\section{Clinical picture of pulmonary plague observed in the paediatric wards of antananarivo}

\author{
Rivo Lova Herilanto Rakotomalala ${ }^{1 *}$, Lovasoa Randriamanga ${ }^{2}$, \\ Mihary Soa Karol ${ }^{3}$, Mioramalala Sedera Aurélien ${ }^{4}$ and \\ Annick Lalaina Robinson ${ }^{1}$
}

\begin{abstract}
${ }^{1}$ Pediatric Ward, University Hospital Mother and Child, Tsaralalana, Antananarivo, Madagascar ${ }^{2}$ Pediatric Ward, University Hospital Soavinandriana, Antananarivo, Madagascar ${ }^{3}$ Neonatal Ward, University Hospital Center for Obstetrical Gynecology Antananarivo, Madagascar ${ }^{4}$ Faculty of Medicine, Antananarivo, Madagascar
\end{abstract}

\section{Abstract}

Introduction: In Madagascar, plague is a highly contagious acute endemic infectious disease. The diagnosis of the most severe form of pneumonic plague remains difficult in children, hence the objectives of the present study; which is to identify the clinical signs of this clinical form in children and to describe its epidemiological and evolutionary profile.

Methods: A retrospective case-control study was conducted in four pediatric wards in Antananarivo during the urban pneumonic plague outbreak from September 2017 to January 2018.

Those cases were defined as children aged 0-15 years old suspected of having plague with positive RDT and PCR, and they were defined as children aged 0-15 years old with negative RDT and PCR.

Results: Fifty-two cases of pneumonic plague were identified, half of which (50\%) were under 24 months of age.

A male predominance was noted with a sex ratio of 1.23 and $86.54 \%$ of the patients were from urban areas.

Several clinical signs were found but none was specific for pneumonic plague: cough $(59.62 \%$ $p$ : 0.5$)$, dyspnea $(3.85 \% p$ : 0.16$)$, chest pain $(3.85 \%$ p: $0.26 \%)$, hemoptysis $(7.69 \% p: 0.17)$, vomiting (9.62\% p: 0.14$)$, diarrhea ( $11.54 \%$ p: 0.45$)$, altered general condition $(38.46 \% p: 0.24 \%)$.

Two deaths were noted (3.8\%).

Conclusion: No specific clinical warning signs have been identified in childhood pneumonic plague. In the event of an epidemic of urban pneumonic plague, any bacterial pneumonia should at least initially include active treatment against Yersinia pestis.

\section{More Information}

*Address for Correspondence:

Dr. Rivo Lova Herilanto Rakotomalala, Pediatric Ward, University Hospital Mother and Child,

Tsaralalana, Antananarivo, Madagascar,

Tel: +261- 344014 923;

Email: lovaherilantoo@yahoo.fr

Submitted: May 01, 2021

Approved: May 12, 2021

Published: May 13, 2021

How to cite this article: Rakotomalala RLH, Randriamanga L, Karol MS, Aurélien MS, Robinson AL. Clinical picture of pulmonary plague observed in the paediatric wards of antananarivo. J Adv Pediatr Child Health. 2021; 4: $046-049$

DOI: 10.29328/journal.japch.1001031

Copyright: () 2021 Rakotomalala RLH, et al This is an open access article distributed under the Creative Commons Attribution License, which permits unrestricted use, distribution, and reproduction in any medium, provided the original work is properly cited.

Keywords: Clinical; Child; Pneumonic plague

A) Check for updates

OPEn ACCEss

\section{Introduction}

\section{Background}

Plague is an acute and highly contagious infectious disease caused by the development in the body of a gram-negative enterobacterium called Yersinia pestis.

It is a zoonosis transmitted to humans by rodents [1-4].

In Madagascar, plague is an endemic disease that mainly affects the central highlands at an altitude of more than $800 \mathrm{~m}$. It presents a seasonal upsurge each year between September and April [5-7].

An epidemic of urban pneumonic plague occurred in Madagascar beginning in late August 2017. Antananarivo was one of the cities which are particularly affected by this epidemic.

Positive diagnosis of pneumonic plague in children 
remains difficult because of the absence of specific clinical signs in children. Severe infections tend to progress rapidly to sepsis. Furthermore, paraclinical diagnostic confirmation is often delayed.

From all the above, it is necessary to rely on epidemiological and clinical clues to rapidly orient the diagnosis of pulmonary and/or septicemic plague in children.

At present, few data are available concerning the clinical picture of pulmonary and/or septicemic plague in children. Several clinical forms have been identified: bubonic plague, septicemic plague and pulmonary plague resulting in pneumonia with severe clinical expression, often responsible for mortality in $50 \%$ of the cases [1,2]. Other forms have also been described: meningeal, pharyngeal, osteoarticular [8-11]. The warning signs may vary according to these different forms, but it is also usual to find atypical clinical signs in young children and infants in different infectious diseases (diarrhea, vomiting or abdominal pain in bacterial pneumonia, acute pyelonephritis or acute meningitis for example).

Thus, the objectives of this study were to identify the signs of pulmonary plague in children and secondarily to describe its epidemiological profile.

\section{Methods}

This was a retrospective case-control study, conducted from September to November 2017 in four pediatric wards in Antananarivo: University Hospital Mother and Child Tsaralalana, University Hospital Joseph Raseta de Befelatanana, University Hospital Mother and Child Ambohimiandra and University Hospital Soavinandriana.

Cases were represented by children aged 0-15 years old with a diagnosis of confirmed pneumonic plague: clinically suspected cases confirmed by both a rapid diagnostic test and PCR or with a positive culture of bronchial secretion or gastric aspirate fluid.

The studied cases were children aged 0-15 years old, who are reported to be suspected plague cases with negative RDT and PCR.

Patients with incomplete clinical data and patients who were discharged prematurely against medical advice were excluded from the study.

Concerning the sample size, the sampling was exhaustive and included all children who met the inclusion criteria during the study period for the cases.

A matching considering the age and gender was done for the controls ( 2 controls for 1 case).

For the following ages:

- $\leq 24$ months: age of controls = age of case $+/-1$ month
- 25 months -5 years: age of controls = age of case $+/-12$ months

- 6 years -15 years: age of controls = age of case $+/-24$ months

Regarding gender, the controls were of the same gender as the case.

The variables studied were: socio-demographic (age, gender, place of residence), disease-related (duration of the stay at the hospital, clinical signs on admission, treatment and course).

All the information was collected on a form prepared for the study, from the admission register and the medical observation record during hospitalization for clinical data.

Data were processed and analyzed by STATA 13 software.

The Chi-square test was used for the study of signs with a significant test for a value of $p<0.05$.

Ethical consideration: the study was started after obtained the authorization of the directors of the establishment and the data collection was carried out with respect for anonymity.

\section{Results and discussion}

On hundred and fifty six patients were included of which 52 cases and 104 witnesses.

Fifty-two cases of pneumonic plague were identified, $51.9 \%$ of which were between 5 and 14 years of age.

A male predominance (57.69\%) was noted with a sex ratio of 1.36 .

Regarding the place of residence, the majority (84.56\%) of the patients were from urban areas.

As for the epidemiological context, we noted a notion of travel to a plague-endemic area in 5 cases.

Generally, the average time from onset to hospitalization was 5.35 days $+/-1.94$.

Figure 1 represents the different clinical signs.

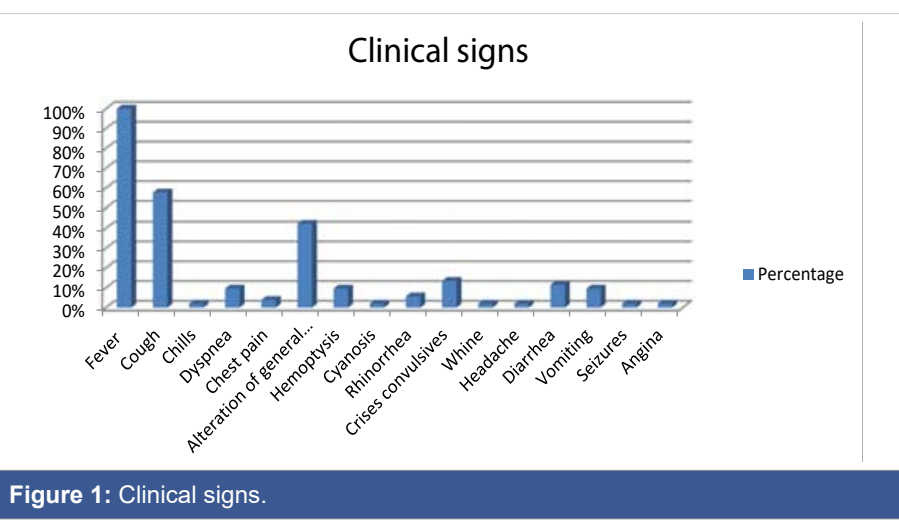


Several clinical manifestations were found, dominated by fever which was present in all patients.

Cough was also present in more than half of the patients $(58 \%)(n=31)$, and an altered general condition was associated in $42 \%$ of cases $(n=20)$.

Hemoptysis was found in $7.69 \%$ of patients $(n=4)$ and dyspnea in $9.61 \%(n=5)$.

Other much less frequent warning signs: digestive (diarrhea $12 \% n=6$, vomiting $10 \% n=5$ ), neurological (seizures $13 \% n=7$, headache $2 \% n=1$ ), ENT (rhinorrhea $6 \%$ $n=3$, angina $2 \% n=1$ ) were noted, chest pain was present in only $4 \%$ of the cases $(n=2)$.

However, none of the clinical signs found in this study were specific for pneumonic plague (Table 1).

The evolution was favorable in the majority of the cases; the death rate was $3.84 \%$.

Fifty-two cases of pneumonic plague were reported in the four pediatric wards of Antananarivo during the study period, which represented $8.71 \%$ of all cases of plague in Madagascar, all ages and forms combined $(n=597)$.

Worldwide, there has been a dramatic decrease in the number of plague cases from 2008 to 2018 from 2683 to 243 cases [12].

In Africa, Madagascar and the Republic of Congo (DRC) are the two countries reporting plague cases: in 2018, 133 cases in DRC.

However, at the global level, no recent data on pneumonic plague in children are available.

According to WHO data in 2015, during the epidemic in Madagascar from September 2014 to February 2015: 261 cases of pneumonic plague were recorded including 8 pediatric cases [13].

\begin{tabular}{|c|c|c|c|c|}
\hline Signs & Cases & Controls & $\boldsymbol{p}$ & OR \\
\hline Fever & 52 & 98 & 0,30 & 0,5 \\
\hline Cough & 31 & 62 & 0,18 & 2,27 \\
\hline Chills & 1 & 1 & 0,56 & 0,37 \\
\hline Alteration of general condition & 20 & 32 & 0,16 & 0,42 \\
\hline Dyspnea & 5 & 10 & 0,55 & 0,26 \\
\hline Chest pain & 2 & 1 & 0,16 & \\
\hline Cyanosis & 1 & 0 & 0,33 & \\
\hline Hémoptysis & 4 & 3 & 0,22 & \\
\hline Rhinorrhéa & 3 & 0 & 0,37 & \\
\hline Headache & 1 & 0 & 0,33 & \\
\hline whine & 1 & 1 & 1 & 0,26 \\
\hline seizures & 7 & 3 & 1 & 0,37 \\
\hline Diarrhea & 6 & 14 & 0,8 & 0,37 \\
\hline Vomiting & 5 & 4 & 0,16 & 0,67 \\
\hline pallor & 1 & 0 & 0,33 & \\
\hline angina & 1 & 0 & 0,33 & \\
\hline
\end{tabular}

According to the literature, bubonic plague is by far the most frequent clinical form, with pulmonary plague being secondary to the spread of the bubonic form [14] but this assumption was disproved by the present study where the pulmonary form increased markedly in number if only for pediatric cases: it increased from 8 in 2014 to 52 .

Fifty-one point nine percent (51.9\%) of patients were between the ages of 5 to 14 years: this result is in line with what has been reported by other authors [7].

However, a non-negligible percentage (48\%) of children aged 13 to 60 months was noted: this is the age group with the most acute respiratory infection in pediatrics [14].

According to this study, the male gender remains the most affected, as has been found in other pathologies where male vulnerability has been noted.

This same finding has also been reported by other authors, particularly concerning plague: male predominance has always been observed (sex ratio 1.47 to 1.36 ) $[7,15]$.

Regarding plague outbreaks, three main outbreaks have been described for Madagascar: rural and urban outbreaks in the Highlands and a coastal outbreak in Mahajanga.

From the first outbreak, especially when hygiene is poor, contagion spreads from person to person, easily aggravated by the movement of people fleeing the epidemic and taking with them those who are already infected $[2,3]$.

In the present study, which was carried out in the capital city, the observed cases came from both urban and rural areas, but the majority of patients lived in urban areas. This can be explained by the precarious hygiene conditions (dirtiness, promiscuity) in the urban areas of the capital, which favor the spread of the disease.

In addition, in cases of plague, the presence of a stay in a plague-endemic area is most often found and helps make a positive diagnosis $[2,3,16]$, which was the case in five patients in this study.

Clinically, patients were hospitalized on average 5.3 days after the onset of the first symptoms.

Infected subjects usually present with an acute febrile state characterized by oscillating high fever [1]; in our case, fever was present in all patients.

Fever was accompanied by an altered general condition in $42 \%$ of the cases: this is a classic association during plague.

However, these two symptoms were not specific warning signs of pneumonic plague in children in this study.

The first symptoms, apart from fever, are essentially respiratory: cough, dyspnea, hemoptysis or purulent sputum $[2,3,13,14]$. 
In our case, cough was the main respiratory warning sign in children, but it was not a specific warning sign in pediatrics either, other signs such as dyspnea and hemoptysis are much less frequent.

Other symptoms were also observed in patients: digestive disorders (vomiting, diarrhea) and neurological (headache, convulsions) and ENT manifestations (angina, rhinorrhea), but none of them were specific to pneumonic plague in children.

Regarding the evolution, it was favorable in the majority of cases in the present study; the pulmonary plague is a lightning form, however the rate of cure is high if it is detected and treated in time $[2,3,13,14]$.

\section{Conclusion}

Several clinical manifestations of pneumonic plague have been found, however, no specific clinical warning signs could be demonstrated in pneumonic plague in children.

Thus, if there is an urban plague epidemic, any bacterial pneumonia should at least initially include active treatment against Yersinia pestis so as not to delay management.

\section{Acknowledgement}

Authors would like to thank all those who contributed to this work: staff in the pediatric ward of the hospital Tsaralalana, Befelatanana, Ambohimiandra, Soavinandriana and people who helped in the realization of this study.

\section{References}

1. French language infectious pathology society. Online Infections. 2001; 1: 8-11.

2. Aubry P. The plague in India. A century after the discovery of the bacillus by A. Yersin. Med Trop. 1994; 54: 289.

3. Epidemiology and management of plague epidemics in the
Mediterranean during the second world war. History of Medecine. 2004.

4. Infectious diseases. Encycl Med Chir. 8-039-V-20. 2001; 8.

5. Ratsitorahina M, Chanteau S, Rosso ML, Randriambelosoa J, Ratsifasoamanana L, et al. Epidémiological aspects of plague in Madagascar. Arch Inst. Pasteur Madagascar. 2002; 68: 51-54. PubMed: https://pubmed.ncbi.nlm.nih.gov/12643093/

6. Chanteau S, Nato F, Migliani R. The value of rapid tests chromatography for the surveillance of epidemic diseases in developing countries by immuno: the example of plague in Madagascar. Med Trop. 2003; 63: 574-576.

7. Pasteur Institute of Madagascar. Plague Atlas in Madagascar, 2004. OMS. The plague. REH. 2005; 80: 138-140.

8. Mann JM, Shandler L, Cushing AH. Pediatric Plague. Pediatrics. 1982; 69: 762-767.

9. Bin Saeed AA, Al-Hamdan NA, Fontaine RE. Plague from Eating Raw Camel Liver. Emerging Infect Dis. 2005; 11: 1459-1457.

PubMed: https://pubmed.ncbi.nlm.nih.gov/16229781/

10. Arbaji A, Kharabsheh S, Al-Azab S, Al-Kayed M, Amr ZS, et al. A 12case outbreak of pharyngeal plague following the consumption of camel meat, innorth-eastern Jordan. Ann TropMedParasitol. 2005; 99:789-793. PubMed: https://pubmed.ncbi.nlm.nih.gov/16297292/

11. Becke TMP, Poland JD, Quan TJ, White ME, MD, et al. Plague Meningitis-A Retrospective Analysis of Cases Reported in the United States, 1970-1979. West J Med. 1987; 147: 554-557.

PubMed: https://pubmed.ncbi.nlm.nih.gov/3424819/

12. Patrice D, Jean-Paul GE. Pilly, Infectios and tropical diseases, Alinéa Plus. 2018; 328.

13. World Health Organization. Plague around the world: $2010-2015 . \mathrm{REH}$. 2016; 91: 89-92.

14. Léon KN, Mbombo D, Nduwa K, Aimé AM, Nathalie KK, et al. Acute respiratory infection and nutritionalstatus in children 0-5 years: case of university clinics of Lubumbashi. Democratic Republic of Congo. Pan Afr Med J. 2014; 19: 393.

15. World Health Organization. Human plague: review of regional morbidity and mortality, 2004-2009. Wkly Epidemiol Rec. 2010; 85: 40-45. PubMed: https://pubmed.ncbi.nlm.nih.gov/20151494/

16. Bulter TH. Plague gives surprises in the first decade of the 21 st century in the United States and worldwide. Am J Trop Med Hyg. 2013; 89: 788-793. PubMed: https://pubmed.ncbi.nlm.nih.gov/24043686/ 